\title{
La décision Nevsun Resources Ltd. c. Araya rendue le 28 février 2020 par la Cour suprême du Canada
}

\section{Renée-Claude Drouin}

\section{(2) OpenEdition}

1 Journals

\section{Édition électronique}

URL : https://journals.openedition.org/rdctss/725

DOI : $10.4000 /$ rdctss. 725

ISSN : 2262-9815

Éditeur

Centre de droit comparé du travail et de la sécurité sociale

\section{Édition imprimée}

Date de publication : 1 avril 2021

Pagination : 152-157

ISSN : 2117-4350

\section{Référence électronique}

Renée-Claude Drouin, «La décision Nevsun Resources Ltd. c. Araya rendue le 28 février 2020 par la

Cour suprême du Canada », Revue de droit comparé du travail et de la sécurité sociale [En ligne], 1 | 2021, mis en ligne le 01 avril 2021, consulté le 01 avril 2022. URL : http://journals.openedition.org/rdctss/ 725 ; DOI : https://doi.org/10.4000/rdctss.725

\section{(c) (†) $\ominus$}

Revue de droit comparé du travail et de la sécurité sociale est mise à disposition selon les termes de la Licence Creative Commons Attribution - Pas d'Utilisation Commerciale - Pas de Modification 4.0 International. 


\section{RENÉE-CLAUDE DROUIN}

UNIVERSITÉ DE MONTRÉAL

\section{LA DÉCISION NEVSUN RESOURCES LTD. C. ARAYA RENDUE LE 28 FÉVRIER 2020 PAR LA COUR SUPRÊME DU CANADA}

Le contentieux relatif à la responsabilité des entreprises pour violation des droits de l'homme dans le cadre de leurs activités à l'étranger évolue progressivement devant diverses juridictions nationales. Les fondements juridiques des recours intentés à l'encontre de sociétés mères dans leur pays d'origine, pour des atteintes commises dans des pays hôtes, reposent fréquemment sur les principes de responsabilité civile, ou de tort dans les systèmes de common law, plus particulièrement sur la responsabilité pour négligence. Le dernier jugement de la Cour suprême du Canada sur le sujet, Nevsun c. Araya, 2020 CSC 5, innove en ouvrant la porte à la reconnaissance d'une cause d'action directe à l'encontre des sociétés pour violation du droit international coutumier. Les opinions des plus hauts magistrats du pays sont toutefois fortement divisées sur la question, qui n'a pas été tranchée de façon définitive puisque le jugement a été rendu au stade préliminaire du recours.

\section{I - LES FAITS DU LITIGE}

Dans l'affaire Nevsun c. Araya, trois travailleurs érythréens allèguent avoir été conscrits dans un régime de travail forcé en Érythrée sur une exploitation minière, copropriété de la société canadienne Nevsun. Ils réclament des dommages-intérêts à cette dernière devant le tribunal de première instance de la Colombie-Britannique, province du siège social et lieu d'incorporation de Nevsun. La mine Bisha où se seraient déroulés les faits allégués appartient à la Bisha Mining Share Company, une société érythréenne, dont les parts sont détenues à $40 \%$ par une société d'État, la Eritrean Mining Corporation, et à $60 \%$ par Nevsun, par l'entremise de filiales. Bisha Mining a sous-traité une partie de la gestion du site à une compagnie sud-africaine (Senet), qui a elle-même conclu des contrats avec deux compagnies de construction érythréennes, l'une contrôlée par l'armée (Mereb) et l'autre par l'unique parti politique du pays (Segen).

Selon les faits rapportés, l'Érythrée a établi un programme de service national auquel sont assujettis tous les Érythréens lorsqu'ils parviennent à l'âge de 18 ans. Le programme comprend une formation militaire de six mois, ainsi qu'une période de service obligatoire de douze mois pendant laquelle les individus sont affectés à des tâches militaires ou encore à « la construction de projets publics d'intérêt national $»^{1}$. C'est dans ce contexte que des conscrits ont été assignés à travailler pour diverses entreprises proches du pouvoir, dont Mereb et Segen. Les demandeurs sont des conscrits qui ont été affectés à la mine Bisha. Ils allèguent avoir été forcés 
de travailler plusieurs années dans des conditions dangereuses; avoir subi des châtiments corporels; avoir été confinés dans des camps de travail sans pouvoir en partir sous peine de représailles; ainsi qu'avoir travaillé de longues heures pour un salaire misérable?2.

\section{II - LES PROCÉDURES}

Les trois travailleurs érythréens ont présenté une demande en dommagesintérêts en première instance en Colombie-Britannique à l'encontre de Nevsun. Les requérants allèguent que cette dernière a commis différents délits de droit interne, dont voies de fait, détention injustifiée, complot et négligence, mais également qu'elle a contrevenu à des normes de droit international coutumier relatives au travail forcé, à l'esclavage, aux traitements cruels, inhumains ou dégradants, et aux crimes contre l'humanité.

Après le dépôt de la demande, Nevsun a présenté différents moyens préliminaires pour faire rejeter le recours. Parmi ces moyens, la demande de rejet du statut de recours collectif pour l'instance déposée par Nevsun a été accueillie, tandis que la demande de rejet de recours fondée sur la doctrine du forum non conveniens a été rejetée. Le pourvoi de Nevsun devant la Cour suprême soulève deux questions distinctes ${ }^{3}$. La première est de savoir si la doctrine de l'acte de gouvernement fait obstacle au recours déposé contre elle en Colombie-Britannique. La deuxième est de savoir si les normes de droit international coutumier invoquées dans les procédures peuvent constituer le fondement d'un recours en dommages-intérêts en droit interne canadien.

\section{III - LE JUGEMENT MAJORITAIRE}

Le jugement majoritaire, rédigé par la juge Abella, comporte une analyse des origines et du développement de la doctrine de l'acte de gouvernement (act of state) en droit anglais. Soulignant qu'il n'y a pas de « définition unique » du concept, la juge Abella reprend les propos de Lord Millet dans l'affaire Pinochet: «la doctrine de l'acte de gouvernement est une règle de droit interne selon laquelle le tribunal national n'est pas compétent pour se prononcer sur la légalité des actes souverains $d^{\prime} u n$ État étranger $»^{4}$. Elle dresse ensuite le portrait des restrictions à l'application de cette doctrine et des exceptions existant dans divers cas.

Le jugement majoritaire souligne toutefois que si le système canadien s'est développé à partir des principes de common law applicables en Angleterre, " le droit canadien a élaboré sa propre méthode $»^{5}$ pour traiter des cas visés par la doctrine de l'acte de gouvernement. C'est par le biais des principes relatifs aux conflits de lois et à la retenue judiciaire que les litiges requérant une évaluation des lois et

$2 \S 11-15$ du jugement.

3 Nevsun n'a pas contesté devant la Cour suprême la décision concernant la non-application de la doctrine du forum non conveniens.

$4 \S 29$ du jugement.

$5 \S 44$ du jugement. 
pratiques d'un État étranger, notamment en matière de droits de la personne, sont juridiquement analysés. Ainsi, « les tribunaux s'abstiendront de tirer des conclusions qui se veulent juridiquement contraignantes pour les États étrangers $»^{6}$. La majorité note que «nos tribunaux prennent en considération la courtoisie, mais comme dans d'autres contextes, le respect découlant de la courtoisie à l'égard des systèmes juridiques étrangers « cesse dès la violation manifeste du droit international et des droits fondamentaux de la personne $»^{7}$. Le jugement majoritaire conclut donc sur ce point que la doctrine de l'acte de gouvernement « ne fait pas partie de la common law canadienne, et ni elle, ni ses principes sous-jacents élaborés dans la jurisprudence canadienne, ne font obstacle aux réclamations des travailleurs érythréens $»^{8}$.

Les juges majoritaires se penchent ensuite sur la demande de Nevsun de radier les réclamations reposant sur les allégations de violation du droit international coutumier. Leurs motifs rappellent que, lorsqu'un tribunal est saisi d'une demande de radiation d'actes de procédure au stade préliminaire, il n'a pas à statuer de façon définitive sur la demande mais simplement à déterminer " s'il est "évident et manifeste" que la demande ne présente aucune possibilité raisonnable de succès " $"$. L'évaluation des chances de succès d'un recours par le tribunal doit tenir compte du caractère évolutif du droit, et donner une ouverture aux nouvelles causes d'action. Pour déterminer s'il existe une cause d'action raisonnable, la majorité de la Cour analyse d'abord si les normes de droit international coutumier invoquées par les demandeurs font partie du droit canadien et, dans un deuxième temps, s'il existe un recours permettant d'accorder une réparation pour la violation de ces normes.

Le jugement majoritaire rappelle que deux conditions sont nécessaires pour la reconnaissance d'une norme de droit international coutumier : l'existence d'une pratique générale et constante, et l'opinio juris (c'est-à-dire le sentiment qu'il existe une obligation juridique $)^{10}$. Au Canada, les normes de droit international coutumier sont intégrées directement en droit interne, sans que cela ne nécessite une intervention législative, par application de la doctrine de l'adoption. Ces normes font donc partie de la common law canadienne et s'appliquent, à moins qu'elles ne soient spécifiquement écartées par le législateur. Selon la majorité, les normes invoquées par les demandeurs - crimes contre l'humanité ; interdiction de l'esclavage; interdiction du travail forcé ; interdiction relative aux traitements cruels, inhumains et dégradants - constituent toutes des normes de droit international coutumier dont elle peut prendre connaissance d'office. Elles font même partie de la catégorie des normes de jus cogens, qui ont un caractère impératif en raison de leur importance fondamentale pour l'humanité ${ }^{11}$.

$6 \S 47$.

$7 \S 50$, citant : R. c. Hape, [2007] 2 R.C.S. 292, § 52.

$8 \S 59$.

$9 \S 64$.

$10 \S 78$ du jugement. Références omises dans la citation.

$11 \S 99$. 
Nevsun allègue toutefois que ces normes ne sont pas applicables directement à son égard puisqu'elle est une société. Les juges majoritaires rejettent cet argument, soulignant que Nevsun "se méprend sur le droit international moderne " $^{12}$. La majorité analyse l'évolution contemporaine du droit international, et sa transition d'un droit centré sur les relations entre États à un droit axé sur la personne humaine, par le biais notamment de l'adoption des grands instruments de protection des droits de la personne. Elle souligne que ces droits dont jouissent les individus doivent être respectés par tous, non seulement les États mais également les acteurs privés, dont les sociétés. Les motifs majoritaires concluent sur ce point qu'en l'absence de conflit avec les lois existantes, "les normes de droit international coutumier invoquées par les travailleurs érythréens font partie de la common law canadienne et sont susceptibles de s'appliquer à Nevsun $»^{13}$.

La Cour analyse ensuite s'il existe un recours civil en droit interne canadien en cas de contravention aux normes de droit international coutumier. La juge Abella rappelle que les principes de common law sont sujets à évolution «lorsque des changements sont nécessaires pour préciser un principe de droit, pour éliminer une incohérence ou pour permettre au droit de suivre l'évolution de la société ». Pour la juge, " la reconnaissance de la possibilité d'un recours pour violation de normes faisant déjà partie de la common law est l'une de ces évolutions nécessaires ${ }^{14}$. La juge souligne l'obligation des États de protéger les individus contre les atteintes aux droits de la personne, y compris celles commises par les sociétés et rappelle l'adage « là où il y a un droit, il y a un recours ». Le jugement majoritaire conclut « qu'il n'est pas évident et manifeste que les tribunaux canadiens ne peuvent élaborer un recours civil en droit interne pour les violations par une société des normes de droit international coutumier adoptées en droit canadien ${ }^{15}$. Les allégations des demandeurs sont donc maintenues.

\section{IV - LES JUGEMENTS DISSIDENTS}

Dans leurs motifs conjoints dissidents en partie, les juges Brown et Rowe expriment leur accord avec le jugement majoritaire en ce qui a trait à l'application de la doctrine de l'acte de gouvernement, mais exposent une opinion divergente quant au rejet des actes de procédure des demandeurs fondés sur des allégations de violation du droit international coutumier. Les magistrats sont d'avis qu'il n'existe pas encore de responsabilité directe des sociétés en droit international coutumier, compte tenu de l'insuffisance de preuve d'une pratique générale et établie des États à cet égard. Au surplus, la doctrine de l'adoption du droit international coutumier en droit interne n'oblige pas l'État canadien à prévoir une réparation civile en cas de violation allant à l'encontre de normes coutumières. La création d'un tel recours ne serait pas du ressort des tribunaux, mais du législateur. En somme, les juges Brown 
et Rowe considèrent que "les motifs des juges majoritaires reposent soit sur des normes de droit international coutumier qui n'existent pas, soit sur l'attribution à la doctrine de l'adoption d'un rôle qu'elle n'a pas ${ }^{16}$.

Dans ses motifs dissidents auxquels souscrit le juge Moldaver, la juge Côté considère que « l'élargissement du droit international coutumier de manière à ce qu'il s'applique aux corporations constitue un écart majeur dans ce domaine du droit». Elle critique le fait que la majorité se fonde sur des " arguments normatifs » plutôt que sur la pratique des États dans ses conclusions sur ce point. Selon elle, les conditions de reconnaissance du droit international coutumier (la pratique et l'opinio juris) ne sont en l'espèce pas remplies. De plus, la juge aurait accueilli le recours de Nevsun sur la base de la doctrine de l'acte de gouvernement. La juge estime que "les recours de droit privé qui reposent sur la conclusion qu'un État étranger a agi de façon contraire au droit international public ne sont pas justiciables ». Toujours selon elle, pour que les demandeurs aient gain de cause il faut que le tribunal reconnaisse que l'Érythrée a agi à l'encontre du droit international public. Une telle situation requiert la retenue judiciaire et en conséquence, la juge aurait rejeté les réclamations dans leur intégralité.

\section{Conclusion}

La divergence entre les motifs majoritaires et dissidents à propos du recours portant sur la violation du droit international coutumier s'explique en grande partie par des visions différentes du rôle du juge dans l'évolution du droit. Pour la majorité, "les tribunaux canadiens, comme tous les tribunaux, jouent un rôle important dans l'évolution continue du droit international ${ }^{17}$ et $" n^{\prime}$ ont aucune raison d'hésiter à mettre en œuvre et à faire avancer » ce droit ${ }^{18}$. Pour leur part, les juges Brown et Rowe considèrent que le pouvoir des tribunaux de faire avancer les règles de droit est limité en ce qui concerne le droit international coutumier : " Ils peuvent reconnaître un changement au droit international coutumier, mais ils ne peuvent eux-mêmes modifier ce droit directement ${ }^{19}$. Pour ces magistrats, les tribunaux élaborent les règles de droit graduellement et doivent hésiter à prendre des trajectoires dont l'issue est incertaine ou inconnue. II n'appartiendrait donc pas aux juges de modifier les limites du droit international.

Les juges majoritaires reconnaissent l'aspect novateur, voire incertain, du recours fondé sur les allégations de droit international coutumier. II leur paraît cependant important que les réclamations soient instruites afin de permettre le cas échéant une éventuelle évolution du droit international des droits de la personne. Au stade préliminaire, les faits sont tenus pour avérés et les allégations de droit sont

\footnotetext{
$16 \S 224$.

$17 \S 70$.

$18 \S 71$.

$19 \S 200$.
} 


\section{CANADA}

maintenues dans la mesure où il n'est pas « évident et manifeste » qu'elles n'ont aucune chance raisonnable de succès. Les demandeurs devront maintenant faire la preuve des faits allégués et démontrer que leurs arguments sont bel et bien fondés en droit. En définitive, il reviendra au juge d'instruction, en première instance, de déterminer si la preuve permet de conclure à une violation du droit international coutumier et le cas échéant quels sont les recours appropriés pour y remédier. 